\title{
Das globale Dorf
}

\author{
Erhard Taverna
}

Die Strategie «Migration und Gesundheit 2002-2006» definiert Interventionen und Zielsetzungen der Bundesämter für Gesundheit BAG, für Flüchtlinge BFF, für Zuwanderung, Integration und Auswanderung IMES und der Eidgenössischen Ausländerkommission EKA. Eingebunden ist auch die Caritas-Fachstelle Gesundheit und Integration in St. Gallen, die im Herbst 2004 mit Fokusgruppeninterviews die Erfahrungen von Migrantinnen und Migranten mit dem Gesundheitswesen in der Ostschweiz untersucht hat. Die wichtigste Botschaft gibt der Arbeit den Titel: «Für mich muss der Arzt fast wie ein Freund sein.» Dabei geht es um gute und weniger gute Hausärzte, mangelnde sprachliche Verständigung, Missverständnisse, stereotype Verallgemeinerungen, rassistische Verhaltensweisen, transkulturelle Kompetenzen und unterschiedliche Therapie- und Rollenerwartungen. Die Studie schliesst mit konkreten Empfehlungen an alle medizinische Fachpersonen, wobei der vertrauensvollen Beziehung zum Hausarzt oder der Hausärztin eine zentrale Bedeutung zukommt.

Die interaktive Informationsplattform www. miges.ch des BAG ist für diese Aufgabe eine weitere empfehlenswerte Hilfe.

Sogar im hintersten Appenzell ist die Welt zu Gast. Einmal jährlich besucht mich der Mann aus Pakistan und ergänzt meinen Notvorrat mit einem Sack Reis. Längst ist er ein erfolgreicher Geschäftsmann und mit einer Schweizerin verheiratet. Turbulent und schweisstreibend war die Beschneidung zweier Brüder vor laufender Kamera eines Onkels, nebst weiteren Familienmitgliedern aus Kosovo. Sie hätten es viel lieber gesehen, wenn ich auf eine Lokalanästhesie verzichtet hätte. Vergnüglich war die Diskussion mit drei Generationen Türkinnen der gleichen Familie über Familienplanung und Sexualität. Wenig erfreulich hingegen die Meldung einer Familie an das Vormundschaftsamt, weil die Mutter um jeden Preis ihre kleine Tochter beschneiden wollte. Bis heute vermisse ich die spanischen Familien, die während der Wirtschaftsflaute nach Galizien zurückgewandert sind. Dafür geniesse ich in jedem Sommer die englisch untertitelten Scharaden fernöstlicher Touristen.
Die meisten Ausländer kommen und gehen, geblieben sind die Frauen aus Thailand und den Philippinen, deren Nachwuchs den lokalen Genpool aufmischt. Allen diesen Menschen verdanke ich, dass meine Arbeit bunter und interessanter ist.

Die Sprache spielt in der Allgemeinpraxis eine untergeordnete Rolle. Oft gleicht ein Mehr an Zeit die fehlenden Kenntnisse aus. Es gibt auch Dorfbewohner, die sich mit Worten schwertun. Mehrmals unterschätzte ich den Einfluss der Verwandten oder den eines traditionell wichtigen Familienmitgliedes. Schwer verdaulich sind die oft ausgeprägten Rollenvorstellungen von Mann und Frau, die traumatisch nachwirkenden Erfahrungen aus dem Herkunftsland oder demütigende Erlebnisse im helvetischen Alltag. Schwierig kann es nach einem abgelehnten Asylantrag werden, wenn Arztzeugnisse zu einer Waffe im Rekursverfahren werden, wenn Sozialansprüche ungerechtfertigt erscheinen oder eine übertriebene Konsummentalität das Vertrauen strapaziert. Nach meiner Erfahrung sind Migrantinnen und Migranten oft sehr gut über unser Gesundheitswesen informiert. Es gibt umfassende und vielsprachige Informationen privater und staatlicher Stellen, aber auch Anwälte, die sich auf diese Assistenz spezialisiert haben.

Die Empfehlungen der Caritas-Fachstelle Gesundheit und Integration kann ich gut annehmen. Im Grunde genommen könnten die Interviews bis auf den Wortlaut auch von alteingesessenen Einwohnern stammen. Auch hier gibt es Fremdsprachige, Randständige, Sozialbezüger, Arbeitslose und vielfältig Behinderte. Auch sie sind manchmal von der Bürokratie überfordert und bringen neben Versicherungspapieren selbst ihr Postcheckbüchlein mit, damit ich ihre Belege dort eintragen helfe. Alle leiden unter dem nasskalten Wetter, das hier endemisch ist. Welcher Abstammung auch immer, alle wünschen eine fachlich kompetente Behandlung in einer freundlichen, einladenden und respektvollen Atmosphäre. Wenn gegenseitig eine Portion Offenheit, Neugier, Geduld und Humor dazukommen, kann es schon fast nicht mehr schiefgehen. 